\title{
A NoSQL Database about Customer Reviews
}

\author{
Paolino Di Felice \\ Dipartimento di Ingegneria \\ Industriale e dell'Informazione, \\ Economia \\ Università di L'Aquila (Italy)
}

\author{
Martina Marinelli, Gaetanino \\ Paolone, Francesco Pilotti \\ Gruppo SI S.c.a.r.l., 64100 \\ Teramo (Italy)
}

\author{
Giovanni Valenza \\ Comit S.r.I., 00193 Roma (Italy)
}

\begin{abstract}
Small and medium-sized enterprises (SMEs) are the backbone of the economy of most countries. There is large evidence in the literature that digitalization improves the market performance of enterprises and, as a consequence, it helps the growth of their businesses. SMEs suffer of budget restrictions, as a consequence within them is low R\&D expenditure as well as the number of employees with IT technical abilities. This lack is an objective obstacle for SMEs to compete in the online market. The aim of the present paper is to reach out a hand to SMEs by presenting in deep details how to structure, load and querying a NoSQL PostgreSQL database being part of the customer service.
\end{abstract}

\section{General Terms}

Data and Information Systems, Digital Systems

\section{Keywords}

Customer service, Complaint, Database, NoSQL, JSON, SME

\section{INTRODUCTION}

There is large evidence in the literature that digitalization improves the market performance of enterprises and, as a consequence, it helps the growth of their businesses. This claim is true in special way for Small and Medium-sized Enterprises (SMEs). Compared with other enterprises, SMEs are confronted with a unique set of issues when competing in the globalised market where operate, in a manner almost hegemonic, giants such as Amazon.

The term "IT readiness" has been introduced in the literature as a precondition that SMEs need to meet in order to fully exploit IT potentials (e.g., [1]). [2] defines SME readiness in terms of enterprise's financial resource to take risks and pressures to change processes, management's IT skills and project support, and employee's IT skills and attitude; while [3] suggests readiness in terms of internal IT infrastructure and number of IT employees. So, different scholars understand the term SME readiness differently; nonetheless, the definitions share the fact that to exploit IT potentials, SMEs must embed "IT-capabilities" in themselves. Unfortunately, for most SMEs this is not true, as stressed, for example, in a 2020 document of the European Commission: "SMEs often must [...] overcome structural barriers such as a lack of management and technical skills [...]" [4]. In light of the findings of [5], a low level of IT readiness within SMEs is a barrier for them to change and grow in the digital-based global market.

The present paper aims at giving an help to SMEs to cover the IT gap. In fact, the paper describes in full details how it is possible to build a database about complaining customers. All firms playing in the market know very well that no matter how hard they try, they cannot please $100 \%$ of the people $100 \%$ of the time, that's why customer complaints are inevitable. Whatever the problem might be, the way they address unhappy customers and handle their complaints can have a major impact on the company's reputation. Obviously, ignoring complaints and failing to resolve them can make the customers leave and spread negative word of mouth. On the opposite, Yilmaz et al. [6] have shown that learning from complaints influence both short- and long-term firm-level performance measures positively. In light of considerations above, SMEs that aim entering the online market of goods and/or services have to adopt a database as the one described in the present contribution.

The paper is structured as follows. Section 2 is about the related work, while Section 3 presents the structure of the database and the SQL scripts for its creation and loading. A meaningful set of query patterns are also given; SMEs can use them to carry out relevant statistics. As stated above, the contribution of this work is the database structure and the query patterns against it; that is the reason why the tables were loaded with a small number of sample data and the query results are not discussed. Section 4 concludes the paper and describes the work in progress at present, as well as the aim of the long term industrial project that has been launched.

\section{RELATED WORK}

No company is so perfect in the delivery of their products/services that dissatisfaction (the source of complaints) does not exist. In this context the well-known saying: "No news, good news" is not always true. So, the correct approach from SMEs that aim playing online lengthily is handling complaints. Handling complaints includes the following three steps: (a) collecting them; (b) analyzing them, and (c) responding appropriately (i.e., overcoming the underlying issue). This paper focuses on the first two steps. Only when a complaint has been captured, the appropriate corrective action can be taken. Nobody complains without reason. No matter how absurd a complaint might sound, it is important to look at the posed issue from the customer's point of view. What might be an effective procedure for resolving customer complaints is a topic outside the scope of the present paper. [7], for example, proposes a seven-step customer complaint procedure. [8] is another interesting, and recent, source for managers to learn about how to deal with customers online complaints. In fact, the study collects best practices to online complaint management from a team of researchers with backgrounds in retailing and relationship marketing.

In [7], authors report that "for every complaint expressed, there are over 25 unregistered complaints. Many dissatisfied customers just quietly take their business elsewhere. [...] Furthermore, a customer with a complaint is likely to tell others about his complaint." Organizations that are truly committed to delivering an effective customer service have to providing them opportunities to complain. A mobile app or a webpage are the easiest way to encourage customers to write 
their comments that, from those collecting points, can then automatically be redirected into a firm's database.

\section{THE NoSQL DATABASE}

The implementation of an effective customer service implies that different channels are adopted in a consistent and coordinated way [9]. A database collecting users complaints is one of those medium. Such a database is a precious intangible assets for firms, since, by querying it, it is possible to extract customers complaints and take appropriate actions to reply to them. In addition, the availability of such kind of data allows the implementation of quantitative methods as an alternative to qualitative ones. In fact, by querying the database over long periods of time it is possible to build statistics useful to get a correct vision of what is going wrong with the offered products and/or services.

PostgreSQL (ver.13) has been adopted as DBMS. The reasons for such a choice are the following. PostgreSQL is an opensource system; using a free software is a unique opportunity for SMEs to keep the costs low. Moreover, PostgreSQL offers a robust support to the JSON data type which, in turn, allows to implement NoSQL databases. The features of NoSQL databases have been reported in the literature (e.g., $[10,11])$. To implement an effective customer service it is highly recommendable that the underlying database is able to host unstructured data.

The customer survey the paper refers to is composed of ten questions. It was taken from page: https://www.surveymonkey.com/mp/customer-satisfactionsurvey-template/.

\subsection{The Tables}

The NoSQL database is composed of five tables: Our_Products, The_Survey_Template, Our_Customers, Sales, The_Reviews. The PostgreSQL's SQL/DDL scripts are listed below.

CREATE TABLE Our_Products (

P_Code serial PRIMARY KEY NOT NULL,

description JSONB);

CREATE TABLE Our_Customers (C_Code serial PRIMARY KEY NOT NULL, Description JSONB);

CREATE TABLE Sales (

P Code integer NOT NULL,

C_Code integer NOT NULL,

"When" date NOT NULL,

PRIMARY KEY (C_Code, P_Code, "When"),

FOREIGN KEY (P_Code) references Our_Products(P_Code) ON UPDATE CASCADE,

FOREIGN KEY (C_Code) references Our_Customers(C_Code) ON UPDATE CASCADE);

CREATE TABLE The_Survey_Template (

TheQuestions JSONB NOT NULL);

CREATE TABLE The_Reviews (

R_Code serial PRIMARY KEY NOT NULL,

C_Code integer NOT NULL,

P_Code integer NOT NULL,

"When" Date NOT NULL,

TheQuestions JSONB NOT NULL,

FOREIGN KEY (C_Code) references Our_Customers(C_Code) ON UPDATE CASCADE,

FOREIGN KEY (P_Code) references Our_Products(P_Code) ON UPDATE CASCADE);

\subsection{A Sample Database}

The database is structured as 30 products, 20 customers, and 100 sales. Five reviews are loaded into The_Reviews table; while the ten questions of the survey are stored into The_Survey_Template table. The PostgreSQL's SQL/DML scripts are listed below. Due to space limits only the INSERT of the first review is shown. Table 1 collects the records about the five reviews. Overall, four different customers wrote, on 2020, five reviews mentioning two different products/services.

Table 1. The tuples in The_Reviews table

\begin{tabular}{|c|c|c|c|}
\hline R_Code & C_Code & P_Code & When \\
\hline 1 & 1 & 3 & '2020-03-13' \\
\hline 2 & 1 & 10 & $' 2020-05-18 '$ \\
\hline 3 & 5 & 3 & $' 2020-04-19 '$ \\
\hline 4 & 10 & 3 & $' 2020-08-04 '$ \\
\hline 5 & 14 & 10 & $' 2020-09-28 '$ \\
\hline
\end{tabular}

Figure 1 shows the screen dump of the created JSONB database and the five tables.

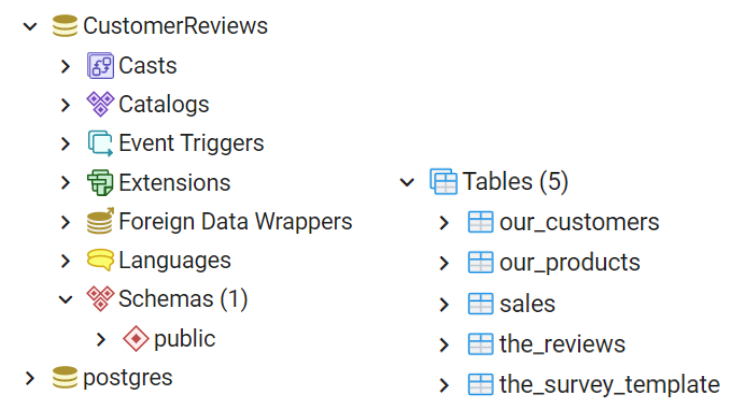

Fig 1: Two screens about the created NoSQL database

-- Insertion of 30 products into Our_Products INSERT INTO Our_Products(P_Code)

SELECT $n$

FROM generate_series $(1,30) n$;

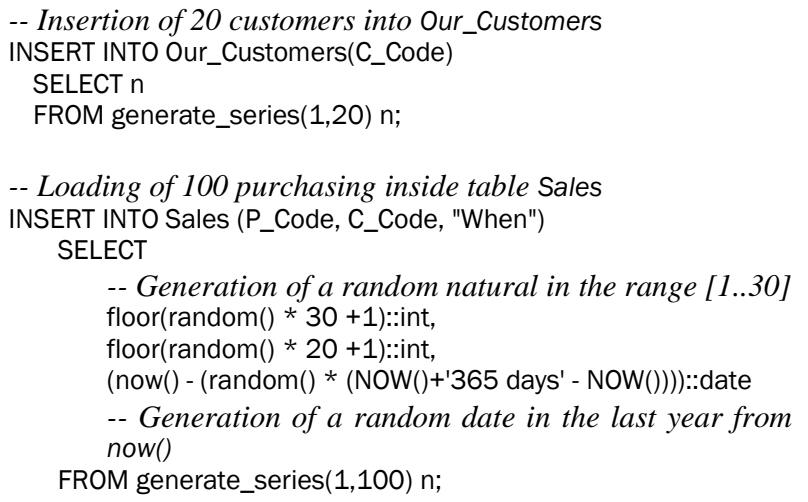

INSERT INTO The_Survey_Template (TheQuestions) VALUES (

'\{"Q1":

\{"description": "Recommend it to a friend. From Not AT ALL (0) to EXTREMELY LIKELY (10)",

"score": 6\}, "Q2":

\{"description": "Overall, how satisfied or dissatisfied are you with our company?",

"field1": "Very satisfied",

"field2": "Somewhat satisfied",

"field3": "Neither satisfied nor dissatisfied",

"field4": "Somewhat dissatisfied", 
"Q3":

"field5": "Very dissatisfied"\},

["description": "Which of the following words would you use to describe our products/services? Select all that apply.",

"field1": "Reliable",

"field2": "High quality",

"field3": "Useful",

"field4": "Unique",

"field5": "Good value for money",

"field6": "Overpriced",

"field7": "Impractical",

"field8": "Ineffective",

"field9": "Poor quality",

"Q4":

"field10":"Unreliable"\},

\{"description": "How well do our product/service meet your needs?",

"field1": "Extremely well",

"field2": "Very well",

"field3": "Somewhat well",

"field4": "Not so well",

"field5": "Not at all well"\},

"Q5":

["description": "How would you rate the quality of the product/service?",

"field1": "Very high quality",

"field2": "High quality",

"field3": "Neither high nor low quality",

"field4": "Low quality",

"field5": "Very low quality"\},

"Q6":

["description": "How would you rate the value for money of the product/service?",

"field1": "Excellent",

"field2": "Above average",

"field3": "Average",

"field4": "Below average",

"field5": "Poor"\},

"Q7":

\{"description": "How responsive have we been to your questions or concerns about our product/service?",

"field1": "Extremely responsive",

"field2": "Very responsive",

"field3": "Somewhat responsive",

"field4": "Not so responsive",

"field5": "Not at all responsive",

"field6": "Not applicable"\},

"Q8":

\{"description": "How long have you been a customer of our company?",

"field1": "This is my first purchase",

"field2": "Less than six months",

"field3": "Six months to a year",

"field4": "1 - 2 years",

"field5": "3 or more years",

"Q9":

"field6": "I have not made a purchase yet"\},

\{"description": "How likely are you to purchase any of our products/services again?",

"field1": "Extremely likely",

"field2": "Very likely",

"field3": "Somewhat likely",

"field4": "Not so likely",

"10".

"field5": "Not at all likely"\},

\});

"Do you have any other comments, questions, or concerns?"

-- Review 1

INSERT INTO The_Reviews (C_Code, P_Code, "When",

TheQuestions)

VALUES

$(1,3$, '2020-03-13',

'\{"Q1": \{"score": 6\},

"Q2": \{"field4": "Somewhat dissatisfied"\},

"Q3": \{"field6": "Overpriced"\},
"Q4": \{"field5": "Not at all well"\},

"Q5": \{"field4": "Low quality"\},

"Q6": \{"field5": "Poor"\},

"Q7": \{"field3": "Somewhat responsive"\},

"Q8": \{"field3": "Six months to a year"\},

\}');

\subsection{Query Patterns}

This subsection collects ten query patterns useful to build statistics on actual data that likely covers a large time interval. For each query the PostgreSQL's screenshot showing the result is also given.

Query 1

Count the number of reviews in the DB (Figure 2).

SELECT Count(*)

FROM The_Reviews;

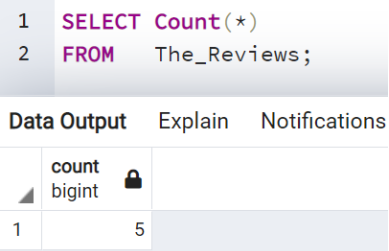

Fig 2: Query 1 and its output

Query 2

Compute the percentage of reviews with respect to the total number of sales (Figure 3).

WITH CTE1 AS

(SELECT Count(*) AS Number_Of_Reviews

FROM The_Reviews)

SELECT ((c.Number_Of_Reviews:::decimal / Count(Sales)) * 100)::numeric(4,2)

FROM Sales, CTE1 AS C

GROUP BY c.Number_Of_Reviews;

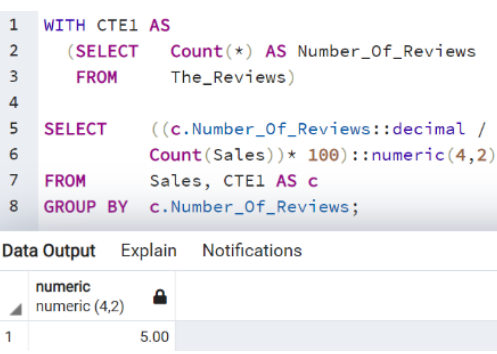

Fig 3: Query 2 and its output

Query 3

Compute the number of distinct complaining customers (Figure 4).

WITH CTE2 AS

(SELECT C_Code

FROM The Reviews

GROUP BY C_Code )

SELECT Count(*)

FROM CTE2; 


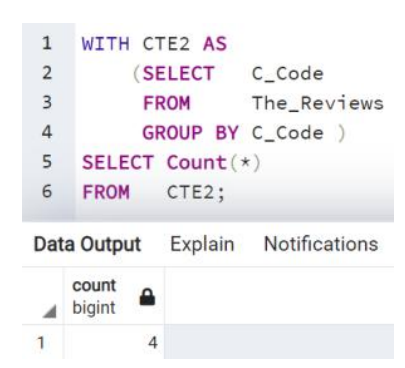

Fig 4: Query 3 and its output

Query 4

Show the C_Code of the complaining customers (Figure 5).

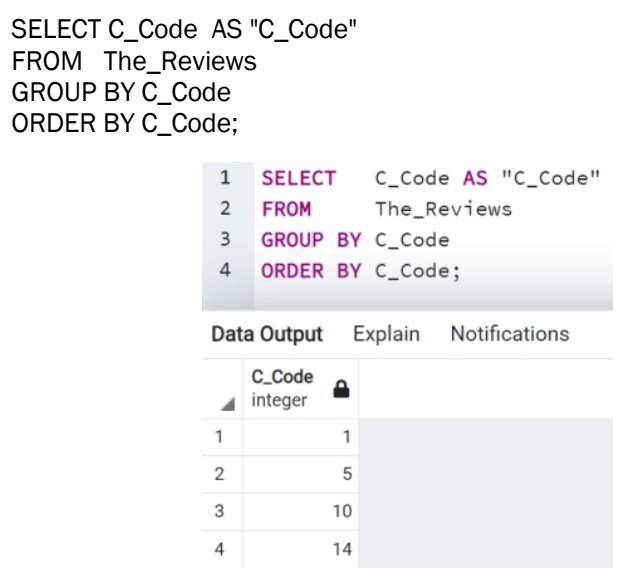

Fig 5: Query 4 and its output

Query 5

Show the C_Code of each complaining customer and the number of reviews he/she wrote (Figure 6).

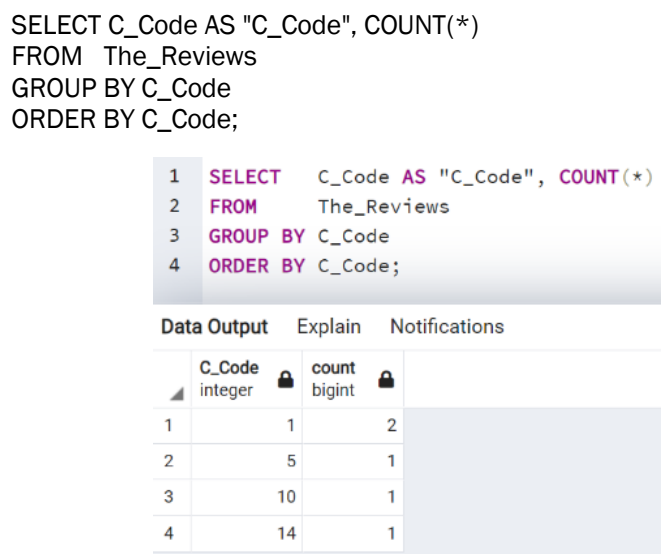

Fig 6: Query 5 and its output

Query 6

Show the overall score of the products mentioned in the reviews (Figure 7).

SELECT P_Code AS "P_Code", TheQuestions -> 'Q1' AS "The score" FROM The_Reviews

ORDER BY P_Code;

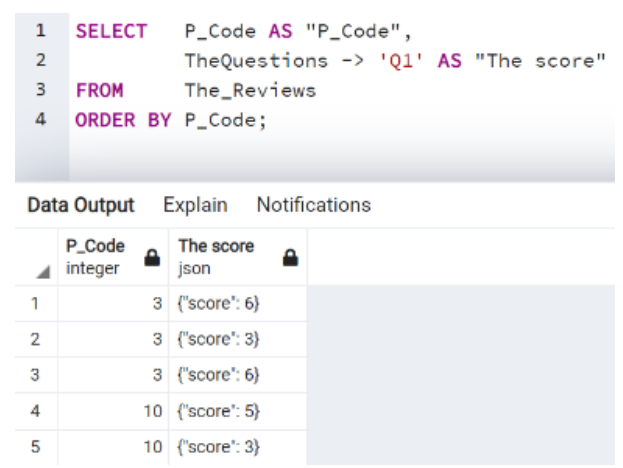

Fig 7: Query 6 and its output

Query 7

Show the P_Code of the products with overall score below 6 (Figure 8).

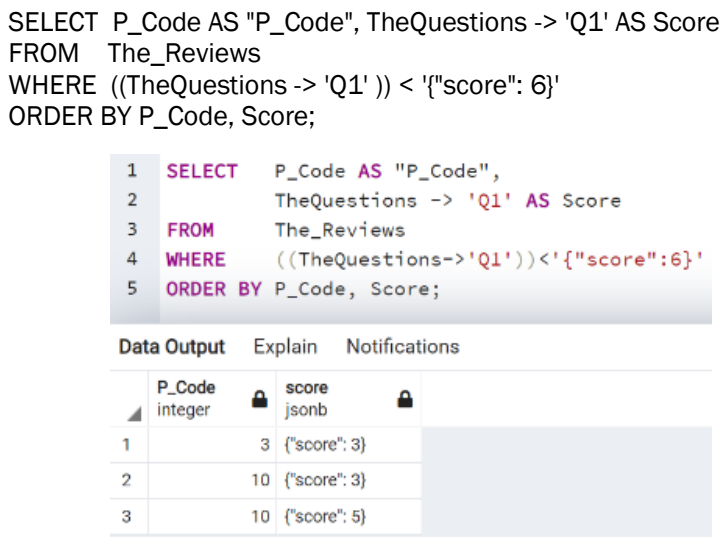

Fig 8: Query 7 and its output

Query 8

Show the P_Code of products with overall score equal to 3 (if any) (Figure 9).

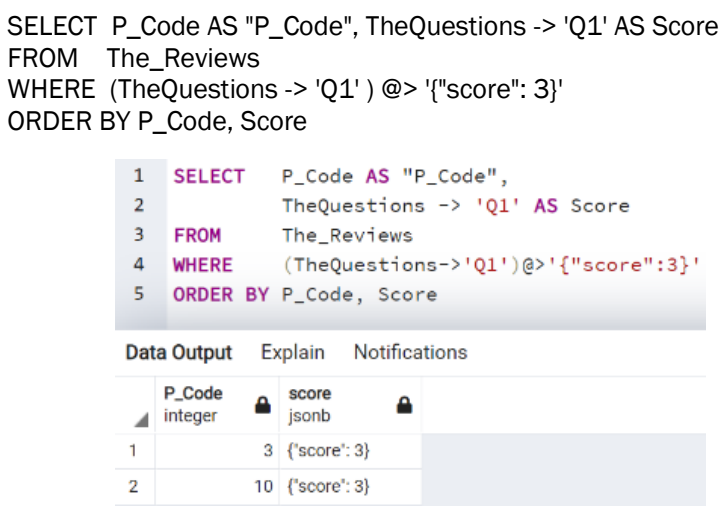

Fig 9: Query 8 and its output

Query 9

Show all the fields of all the reviews in the DB mentioning a given product (e.g., P_Code=10) (Figure 10).

SELECT jsonb_each(TheQuestions) AS "The answers" FROM The_Reviews WHERE P C Code $=10$

The output of this query is composed of as many rows as the number of questions in the survey times the number of tuples which have P_Code=10.

SELECT to_jsonb(TheQuestions) AS p 
